\section{Maria Teresa Calapso's Hyperbolic Pythagorean Theorem}

The Mathematical Intelligencer encourages comments

about the material in this issue. Letters to the editor

should be sent to either of the editors-in-chief, Chandler

Davis or Marjorie Senechal.

7 read with interest Paolo Maraner's recent Mathematical Intelligencer note "A Spherical Pythagorean Theorem" (Vol. 32, No. 3, Fall 2010, 46-50, DOI:10.1007/s00283010-9152-9). In it, the author shows that a proper generalization of the Pythagorean theorem that would render it true in an absolute setting, cannot stay with the hypothesis that one angle of the triangle be right, but rather has to relax it to state that one angle, say $A$, should be the sum of the other two, say $B$ and $C$. Also the Pythagorean theorem should state that the areas of the circles with sides $b$ and $c$ as radii should be equal to the area of the circle with side $a$ as radius (let us denote the latter area by $\bigcirc a$ ). I would like to point out that precisely this form of the Pythagorean theorem was stated and proved in the hyperbolic plane by Maria Teresa Calapso in [2], where it is shown that the converse holds as well, that is, that we have $\bigcirc a=\bigcirc b+$ $\bigcirc c$ only in triangles in which $A=B+C$ holds. In [8] it was shown that the generalized Pythagorean formula, valid in any hyperbolic triangle, is $\bigcirc a=\bigcirc b+\frac{\sin (A-B)}{\sin C} \bigcirc c$, and $[1,3,7]$ contain like-minded forms of the generalized Pythagorean theorem. As its title indicates, the main novelty in Paolo Maraner's paper is the fact that this version of the Pythagorean theorem holds in the spherical setting as well. Even in the hyperbolic case, the paper has the merit of providing a short proof that appeals only to basic formulas of hyperbolic geometry, simpler than the proofs in $[1,2]$.

The author also asks for the proper axiomatic setting in which the above-stated Pythagorean theorem would hold. Given that areas of circles demand the full axiomatic import of the real numbers, the version referring to areas of circles falls outside elementary (first-order logic) axiomatic considerations. If a version regarding triangles satisfying $A=$ $B+C$ turns out to be true in Hilbert's absolute geometry (axiomatized by the axioms I 1-3, II, and III of [5] or by the axioms A1-A9 of [6]), then it must involve areas of polygons on the sides $a, b, c$ (perhaps right isosceles triangles with equal sides having the length of the triangle side on which they are erected), with the Hilbert definition of area equality as equivalence by completion (Ergänzungsgleichbeit).

Relevant for an absolute version of the Pythagorean theorem is also the absolute version of the Intersecting Chords Theorem (III.36 in Euclid's Elements) in [4].

\section{REFERENCES}

[1] Familiari-Calapso, M. T., Le théorème de Pythagore en géométrie absolue. C. R. Math. Acad. Sci. Paris. Sér. A-B. 263 (1966), A668A670.

[2] Familiari-Calapso, M. T., Sur une classe di triangles et sur le théorème de Pythagore en géométrie hyperbolique. C. R. Acad. Sci. Paris Sér. A-B 268 (1969), A603-A604.

[3] Calapso, M. T., Ancora sul teorema di Pitagora in geometria assoluta. Atti Accad. Peloritana Pericolanti Cl. Sci. Fis. Mat. Natur. 50 (1970), 99-107.

[4] Hartshorne, R., Non-Euclidean III.36. Amer. Math. Monthly 110 (2003), 495-502.

[5] Hilbert, D., Grundlagen der Geometrie, 12. Auflage. Teubner, Stuttgart, 1977.

[6] W. Schwabhäuser, W. Szmielew, and A. Tarski, Metamathematische Methoden in der Geometrie. Springer-Verlag, Berlin, 1983.

[7] Vrănceanu, G., Sopra la geometria noneuclidea. Atti Accad. Peloritana Pericolanti Cl. Sci. Fis. Mat. Natur. 50 (1970), 119-123.

[8] Vrănceanu G. G., Sur la trigonométrie noneuclidienne. Rend. Circ. Mat. Palermo (2) 20 (1971), 254-262

Victor Pambuccian

Division of Mathematical and Natural Sciences

Arizona State University-West Campus

Phoenix, AZ 85069-7100

USA

e-mail: pamb@asu.edu 